zur Fixierung von Indanthrenfärbungen dar. Nach Aufdruck der Farbe wird gedämpft, dann in $20^{\circ} \mathrm{Be} \mathrm{NaOH} 70-80^{\circ} \mathrm{C}$ warm abgezogen. Die Umwandlungsprodukte des Indigos gehen in Lösung, während die mit in der Druckfarbe enthaltenen Indanthrenfarben fixiert werden. Man hat hier den interessanten Fall des Ersatzes eines Küpenfarbstoffes durch einen anderen vor sich. Es scheint, dab die Adsorption der bekannten Anthrachinonderivate energischer vor sich geht als die des Indigos. Jedenfalls wird hier eine Adsorptionsverbindung zugunsten der Bildung einer underen gelöst. Man hat allerdings zu bedenken, daß das im Dampfe mit Rongalit und Leukotrop mit. Hilfe von Zinkoxyd gebildete Umwandlungsprodukt des Indigos zur. Faser nicht mehr die Affinität wie der unveränderte Farbstoff zeigt.

Ueber die Veränderung der tarbenden Eigenschaften der Wollfaser schreibt ein mit G. P. signierender Verfasser in -Oesterreichs Wollen- u. Leinen-industrie " 1916 , S. 124. Wolle erlangt durch die Behandlung mit Gerbstoffen und nachträgliche Beliandlung in Metallsaizen die Eigenschaft, dats sie sich auch in basischen Farbstoffen gut färbt und die Affinităt zu sauren Farben verliert.

\section{Appretur und Impragnierung.}

E. Kraus bearbeitet die Verwendung basisch schwefelsaurer Tonerde in der lmprägnierungstechnik und im Zeugdruck. Er kommt zu dem Schlusse, daß zum Wasserdichtmachen der Stoffe nur sulfatfreie, essigsaure Tonerde oder ameisensaure Tonerde zulässig sei. Die Anwesenheit von Suliaten selbst in geringen Mengen, schwächt beim Imprägnieren die Faser beträchtlich. Auch in anderer Hinsicht wird der Gehalt an Sulfaten vermieden werden müssen; es hat sich nämlich nach Versuchen des Verfassers gezeigt, dab bei Sulfaten eine Nachbehandlung in alkalischen Flüssigkeiten nötig ist, ưn möglichst die ganze Tonerde auf der Faser zu behalten, aufierdem wird aus den Lösungen basischer Tonerdesalze Tonerde in kolloider Form von der Faser adsorbiert. Halle r) zeigie seinerzeit, daB Sulfate in Lösungen von Tonerdesalzen die hydrolytische Spaltung hintanhalten, es daher nötig sei, bei Anwendung derartiger Beizen eine alkalische Passage folgen zu lassen, um möglichst viel Tonerde auf der Faser zu fixieren. Diese Passage ist nicht notwendig bei Lösungen basisch essigsaurer Tonerdesalze, bei denen die Tonerde in kolloider Form auf der Faser befestigt bleibt. Es würde sich also auch zu Imprägnierungszwecken die Anwendung dieser Form von Tonerdesalzen empfehlen. (FärberZtg. 1916, S. 241.)

Hermann Dutschke, Elberfeld, beschreibt im D. R. P. 285023 ein Verfahren zur Erzeugung von Glanz auf baum wollenen Waren. Die Stoffe werden mit Lossungen von Salzen, z. B. $\mathrm{Na}_{2} \mathrm{SO}_{4}, \mathrm{CaCl}_{2}$, $\mathrm{Na}$-Azetat u. dergl. imptägniert, dann heiB kalandert. Dadurch werden die sich aus den Salzen bildenden Kristalle in den Faden hineingepreBt und erzeugen reflektierende Flächen, ähnlich der bekannten Riffelung mit gravierten Stahlwalzen. Jedenfalls hat man bei dem Verfahren dafür zu sorgen, dab Salze verwendet werden, die bei der heißen Kalandrierung nicht Säuren abspalten, will man nicht die Festigkeit der Ware aufs Spiel setzen.

1) Farber - Ztg. 1912, 489 .

\title{
Ueber die kolloide Natur des Kolophoniums, I.
}

Von Ludwig Paul (Strabburg i. E.).

(Eingegangen am 19. Soli 1917.)

Das Kolophonium ist, wie die meisten Harze, noch niemals Gegenstand kolloidchemischer Untersuchungen gewesen, und daher auch in dem groBen Sammelwerk von A. T s ch i r ch') die kolloide Natur desselben mit keinem Wort er"ähnt. Auch Wo. Ostwaldz) in seinem, die modernen Anschauungen der Kolloidchemie in überaus klarer und umfassender Weise reflek-

1) T sch irch, Harze und die Harzbehälter (1906).

3) Wo. Ostwald, Die Welt der vernachlässigten Dimensionen (Dresden und Leipzig 1916), 111. tierenden Werk, streift die Harze nur im Vorübergehen und rechnet sie den Isodispersoiden $2 u$.

Erst die im Jahre 1913 von mir begonnenen Untersuchungen: Ueber wasserlösliche Harzsäuren, die in einer Reihe von Aufsätzen in der Chemischen Revue über die Fett- und Harzindustrie im Jahre 1914 erschienen, steilten auf S. 80 cinen Vergleich mit der Stärke an. Dann spăter im Jahre 1915 wurde in der. SeifensiederZeitung S. 237 die große Absorptionsfähig- 
keit der Kolophoniumsubstanz für Wasser bzw. doren Vermögen, Wasser kolloid zu binden, hesvorgehoben, und damit die kolloide Natur als Grundlage für alle am Kolophonium za beobachtenden, seine rätselhafte: Natur bedingenden Erscheinungen, festgesteilt. Inzwischen haben sich die kolloiden Erscheinungen an dieser Substanz in erstaunlicher Fülle vermehrt, so dab es geboten erschien, diese in einer zusammengefabten Darstellung wach einem Primzip zu beschreiben und damit gleichsam den Platz zu belegen der dem Kolophonium als organische Kolloidsubstanz zukommt.

Der kolloide Zustand ist ein allgemein unöglicher Zustand der Materie (Ostwald S. 20) und das auf S. 15 aufgestellte Schema der dispersen Systeme zeigt allgemein den Weg an, auf dem ein gegebener Stoff in den kolloiden Zustand übergetührt werden kann.

Aber das trifft für eine Reihe namentlich organischer Kolloidsubstanzen, wie Leim, Eiweif usw. nicht $z u$, da diese sich bereits in dem kolloiden Zustand befinden, der gewissermaßen der Ausdruck bzw. die Erscheinungsform ihrer an und für sich rätselhaften Materie ist. Noch mehr trifft letzteres beim Kolophonium zu, dessen Ausnahmestellung noch dadurch erhöht wird, daß es kolloide Lösungen, wie Leim, Eiweik; oder den Uebergang eines zunehmenden Dispersionsgrades von groben Zerteilungen zu kolloiden Lösungen, wie Gold usw., nicht zeigt. Wenn nach einem Analogon gesucht wird, das hinsichtlich der äuBeren form wenigstens dem Kolophonium in seinem kolloiden Verhalten an die Seite gestellt werden kann, so ist es die von Wo. Ostwald auf S. 122 anschaulich geschilderte Kieselsäure, die in kristallisierter wasserfreier Form als Quarz auftritt, dann aber in einer Reihe von Substanzen, die bei abnehmender kristallinischer Struktur eine Wasseraufnahme bis zu 30-. 40 Prozent zeigen, un schlieflich in das amorphe, aus einer alkalischen Kieselsäurelösung gefällte Laboratoriumspräparat, das sogenannte normale Kieselsäuregcl, überzugetien. Danit ist eine Dispersoidreihe gegeben, die den Uebergang von grobdispersen, makroskopischen Kristallen bis zu typisch kolloiden Tellchen zeigt.

Das trifft äuBerlich berrachtet durchaus für die Kolophoniumsubstanz $2 u$, wenn die später zu beschreibende kristallisierte $\not$-Abietinsüure als absolute Kolophoniumsubstanz aus ibrer alkalischen Lösung gefält wird und dadurch in kolloide $\gamma$-Pininsäure umgewandelt wird, oder umgekehrt letztere durch wasserentziehende
Mittel in allmählich immer kristallinischer werdende Produkie, die schließlich in die kristallisierte $\gamma$-Abietinsäure übergehen, übergeführt wird.

Diese vorerwähnten Beziehungen lassen es unzweifeihaft erscheinen, dab das Kolophonium den festen kolloiden Lösungen (Wo. Ostwald S. 120) zugerechnet werden muB.

Diesen aber und auch allgemein den Gelen gegenüber zeigt das Kolophonium charakteristische Unterschiede, die es zu cinem neuen Typus fester kolloider Losungen stempeln.

Wird eine alkalische Lösung des Kolophoniums mit Salzsäure versetzt, so wird gleichzeitig ein stöchiometrisch immer gleichbleibender Teil des Dispersitätsmittels mit gefällt und 2war in hochdisperser Verteilung, die der molekularen wenn nicht gleich, doch annähernd gleich kommt, so dab eine nahezu molekuiare Vereinigung der hochdispers ausfallenden Kolophoniumsubstanz mit seinem ebenso hochdispers fallenden Dispersitätsmittel entsteht. die einer chemischen Verbindung wohl ähnlich ist, aber einer solchen nichit gleichkomnt. Denn alle hierfür als Ausgangssubstanzen zu wählenden Präparate : Kolophoniumstücke, $\gamma$-Pininsäure, Sylvinsäure, Malysäure und $\gamma$-Abietinsäure sind chemisch gleichwertig und alle geben aus alkalischer Lösung gefallt die amorphe, in hochdisperser Verteilung mit ihrem Dispersitătsmittel sich befindliche $\gamma$-Pininsäure, die ihrerseits stets denselben Schmelzpunkt $\left(75-76^{\circ}\right)$ zeigt. Wäre das Wasser chemisch gebunden, so müßte damit ein Gleichgewichtszustand eingetreten sein. Das aber ist nicht der Fall.

Auf Grund des Wenzel'schen Gesetzes nimmt die Reaktionsgeschwindigkeit zwischen festen und flüssigen. Stoffen proportional der Berührungsfläche zu (Wo. Ostwald S. 60). Schon die Reaktionsfähigkeit e in es mit ungeheurer Oberfläche versehenen kolloiden Systems erscheint demnach gesteigert; sie wird sich bei gleichzeitiger Anwesenheit eines zweiten kolloiden Systems mit hohem Dispersitätsgrad, in diesem Falle des gefällten Dispersitätsmittels, zu einer gegenseitigen chemischen Einwirkung steigern, die $2 u$ einer allmählichen, aber vollständigen Umwandlung der ursprünglichen Kolophoniumsubstanz führt. Das Wesentliche und Charakteristische des Kolophoniums liegt also nicht nur in der Pähigkeit, eine stets dem Gewicht nach gleichbleibende Menge seines Lösungsmittels zu fällen und zur kolloiden Verteilung $\mathrm{zu}$ bringen und vor allem auch fest- 
zuhalten, sondern auch darin, hiermit die Grundlage für eine spätere gegenseitige Einwirkung zu schaffen : dabei wird der Schmelzpunkt der als Ausgangssubstanz gewählten $\gamma$-Abietinsäure von von $161-163^{\circ}$ auf $75-76^{\circ}$ heruntergedrückt, den hohen Dispersitätsgrad der neu entstandenen, den ersten kolloiden Zustand darstellenden $\gamma$-Pininsäure, anzeigend, der allerdings auch durch das Wasser an sich stark beinflußit, d. h. erniedrigt erscheint.

Wenn man sich auch hinsichtlich der form, in der Wasser in der Kolophoniumsubstanz gebunden ist, keine klare Vorstellung machen kann, so viel steht fest, daf es auf keine Weise entfernt werden kann, ohne die Substanz größtenteils zu zerstören.

Dies alles zusammengenommen, zeigt das Kolophonium allen anorganischen und organischen kolloiden Substanzen gegenüber wesentliche Unterschiede, und stellt sich daher als neuen Typus einer festen kollöiden Lösung dar, deren geschmolzene Form die im Handel befindliche Substanz, sowohl das braunrot gefärbte, eine durchscheinende Masse bildende amerikanische, wie das hellgelbe, auch durchsichtige spanische (französische?) ist.

Die kolloiden Eigenschaften bleiben der Substanz erhalten, wenn diese mit Hilfe der Kolophonium-Harzseife in die ein feines weikes amorphes Pulver bildende Hauptsubstanz, in die $\gamma$-Pininsäure, übergeführt wird. Diese ist in dem Kolophonium bis zu 90 Proz. enthalten und besitzt einen Schmelzpunkt von ca. $75-76^{\circ}$, welcher mit demjenigen der. Handelsware übereinstimmt, der für die feingepulverten großen und durchsichtigen Stücke bei $74-76^{\circ}$ festgestellt wurde. Wird ein Teil der letzteren in eine kochende Sodalösung, aus einem Teil Ammoniaksoda und ca. 201/2 - 30 Teilen Wasser bestehend, eingetragen, so findet zwar eine sichtliche Reaktion, aber keinerlei Kohlensäureentwicklung statt. Die saure Natur der Kolophoniumsubstanz oder der dieser gleich zu setzenden $\gamma$-Pininsăure ist so gering, dab sie nicht einunal Kohlensäure aus den Karbonaten zu verdrängen vermag, sondern sich wahrscheinlich begnügt, diese in die sauren Salze zu verwandeln. Beim Erkalten scheidet sich die Kolophon-Natronseife oder wie sie später genauer, um sie von anderen Harzseifen zu unterscheiden, als $\gamma$-Natronharzseife bezeichnet wurde, fast vollständig aus, infolge ihrer Schwerlöslichkeit in ca. $4-5$ prozentiger Sodalösung. Durch diese Behandlung ist die Kolophoniumsubstanz in zwei Teile zerlegt worden. Außer der bis 175 Proz. der angewandten Kolophoniumsubstanz ausmachenden, sich als schmierseifenähnliche Masse abscheidenden $\gamma$-Natronharzseife, die am besten auf einem Nesselfilter gesammelt wird, enthalt das stark sodahaltige Filtrat die leichter löslichen Substanzen, die auf Zusatz von Salzsäure gefällt werden und als KL.-Substanz nicht nur die schon vorher in Kolophonium vorhandenen, sondern auch die bei der Behandlung mit kochender Sodalösung entstehenden Substanzen enthalten. Aus der abgeschiedenen $\gamma$-Natronharzseife, die vorteilhaft in einer gefärbten, mit Gummistopfen verschlieBbaren Glasflasche aufbewahrt wird und durch häufiges Schütteln von dem letzten Rest der Mutterlauge befreit werden kann, erhält man die $\gamma$-Pininsäure vorn Schmelzpunkt 75 bis $76^{\circ}$, wenn deren wässerige Lösung mit Salzsäure gefällt wird. Da dieselbe Kochsalz und Chlorkalzium zu absorbieren vermag, ist ein nicht zu übertreibendes Auswaschen nur mit destilliertem Wasser bis zur Entfernung der Mineralsäure zulässig. Die abgequetschte oder auch abgepreBte Substanz darf nicht dutch Erwärmen getrocknet werden. Die Ausbeute der lufttrockenen $\gamma$-Pininsäure betrăgt ca. 90 Proz. des angewandten Kolophoniums und zeigt den richtigen Schmelzpunkt $75-76^{\circ}$. Wie bei den meisten Harzsubstanzen, sind auch hier zwei Schinelzpunkte zu beohachten. Der erste liegt bei $70-71^{\circ}$, wobei die Substanzprobe eine durchscheinende, homogen zusammengeschmolzene Masse bildet; der zweite $\left(75-76^{\circ}\right)$ ist an der Bildung einer aus durchsichtigen Tropfen bestehenden Tropfenkette erkenntlich. Bei der Elementaranalyse wurden $C=77,97, H=$ $9,61, O=12,42$ gefunden. Beide Substanzen, die $\gamma$-Natronharzseife sowohl, wie die $\gamma$-Pininsäure sind nicht unzersetzt aufzubewahren; namentlich letztere zeigt schon nach einigen Wochen durch einen sich immer mehr steigernden Schmelzpunkt eine fortlaufende Zersetzung an, die die Substanz unter dem Einflub des kolloid gebundenen Wassers erleidet.

Erscheint durch die vorher beschriebene alkalische Behandlung die kolloide Natur des Kolophoniums in keiner Weise gestört, im Gegenteil, wird diese erneut den davon befreiten Substanzen, wie an der später zu erörternden $\gamma$-Abietinsäure $2 u$ zeigen ist, durch alkalische Behandlung wieder zugeführt, so übt eine Behandlung mit Alkohol die gegenteilige Wirkung aus. $\mathrm{Da}_{\mathrm{a}}$ nun in den meisten Fällen zur Trennung der namentlich im amerikanischen Kolophonium enthaltenen Bestandteile: Alkohol ver- 
wand wird, so ist es weiter nicht verwunderlich, daf infolge der wasserentziehenden Eigenschaften des Alkohols die große Adsorptionsfähigkeit des Kolophoniums zerstört ivird und damit deren Fähigkeit, das Wasser kolloid zu binden.

Von dem Bestreben geleitet, die ursprünglich im Kolophonium anzunehmende amorphe Substanz durch eine Alkoholbehandlung zu reinigen und eine neutrale indifferente Harz" substanz zu entfernen, deren Vorhandensein die amorphe Kolophoniumsubstanz hindere, kristallinisch bzw. kristallisiert aufzutreten, hat man der Natur des Kolophoniums Gewalt angetan. Schon vor $80-90$ Jahren glaubte Unverdorbe ${ }^{3}$ ) (1824-1832), die von ihn gefun. dene amorphe Pininsäure durch ihre Leichtlosslichkeit in Alkohol von der schwerer löslichen und kristallinischen Syivinsäure trennen zu müssen. Auf dieser Grundlage sind schlieBlich alle weiteren Arbeiten, auch diejenigen Ts ch i r ch's (S. 650), aufgebaut, da es belanglos ist, wenn $\mathrm{Maly}$ an die Stelle der kristallinischen Sylvinsäure die bei $135^{\circ}$ schmelzende, aber besser kristallisierte Abietinsäure (sogen. Maly-Säure, Tschirch S. 663) setzt, die von Tschirch mit Hilfe seiner rationell aufgebauten Ausschüttelungsmethode in drei, von ihm für Isomere gehaltenen, $\alpha$-, $\beta$ - und $\gamma$-Abietinsäuren zerlegt werden kann, deren Schmelzpunkte allerdings ziemlich nahe, bei ca. $155^{\circ}$, zusammenfallen ${ }^{4}$ ).

Die Bedeutung der Arbeiten Unverdorben's, der als der erste eine amorphe Harzsäure, die Pininsäure und deren Umwandlungsfähigkeit in die kristallinische Sylvinsäure mittelst Alkohol erikannte, bleibt auch dann erhalten und grundlegend, wenn diesen Beobachtungen eine falsche Auslegung gegeben wurde. Denn die Pininsäure und die Sylvinsäure werden nicht voneinander getrennt, ebensowenig ein von fast allen Forschern beobachtetes neutrales und indifferentes Harz entfernt, um die Pininsäure in die Sylvinsäure oder in die Malysäure bzw. in die drei Isomeren $\alpha$, $\beta$ - und $\gamma$-Abietinsäuren zu verwandeln. sondern es verliert die Pininsäure durch die Alkoholbehandlung allmählich ihre kolloide Fkhigkeit und verwandelt sich dabei zunächst in die mit geringerer kolloider Fähigkeit behafteten, nunmehr kristallinischen Sylvin- und Malysüuren, um schlieblich in die

3) Tschirch, Die Harze und die Harzbehalter $(1906), 626$

4). Tschirch, Die Harze und die Harzbehälter (1906), 660 . deutlich kristallisierte Abietinsäure überzugehen, die, wail ihr keine Spur eines kolloiden $\mathrm{Zu}$ standes mehr anhaftet, als absolute Kolo. phoniumsubstanz zu bezeichnen ist. Diese ist wasserfrei und unbegrenzt hinsichtlich der Zeit aufzubewahren, weil das zersetzende, kolloid gebundene Wasser fehit.

Die Beobachtungen Unverdorben's konnte ich bis auf das neutrale indifferente Harz bestätigen und erachte es als selbstverständlich, daß der Name Pininsäure für die amorphe und eigentliche Grundsubstanz erhalten bleibt.

Auf Grund der vorher angedeuteten Arbeiten hat sich die Vorstellung über die Natur des Kolophoniums nicht von der Annahme eines indifferenten Harzes trennen können, in dem die hochschmelzenden Abietinsäuren gewissermaßen eirigebettet oder gelöst seien.

Der Irrtum, dem alle Forscher auf diesem Gebiete verfallen sind, erklärt sich aus der einseitigen Stellung, mit der sie das Kolophonium mehr als chemische Substanz betrachteten und dessen physikalische Natur auBeracht lieben, die nur amorphe, mit kolloid gebundenem Wasser behaftete Substanzen kennt, die bei der Behandlung mit Alkohol zum Teil schmierenhaft werden, aber nichts weiter sind als eine durch Wasser und namentlich durch Alkohol flüssig gewordene kolloide Lösung. Namentlich gilt dies von der von Tschirch benutzten Ausschüttelungsmethode, die nur dann rationell erscheint, wenn, wie zu erwarten war, diese Schmieren bei der Behandlung einer ätherischen Kolophoniumlösung der Reihe nach mit einprozentiger Ammoniumkarbonat-, dann Soda- und schließlich Kalihydratlösung, vermieden wurden. Dies geschah aber nicht; da sich aus den bei der Behandlung mit einprozentiger Ammoniumkarbonatlösung erhaltenen Substanzen durch Alkohol nach einigem Stehen reichlich braune Schmieren abschieden (Tschirch S. 651). Wie sollte auch ein neutrales und indifferentes Harz selbst bei 600 maliger Ausschüttelung mit einprozentiger Ammoniumkarbonatlösung in so reichlicher Menge in Lösung gehen? Die Bildung der bei der Alkoholbetandlung gleichzeitig beobachteten Kristalle geschah nur durch Wasserabgabe, und der dadurch entstandene verdünntere Alkohol bildete mit dem Rest der Substanz eine kolloide Lösung, die nur einzutrocknen braucht, wozu allerdings eine lange Zeit gehört, um wieder brauchbare Ausgangssubstanz zu sein; noch besser ist eine eineute Lösung in Alkali. Von der Richtigkeit meiner Auffassung kann man sich leicht durch Ueber- 
gieben einer breiigen Paste der. anfangs beschriebenen $\boldsymbol{\gamma}$-Pininsäure mit Alkohol überzeugen. Diese flieBt dabei vollständig zusammen und bildet eine leicht knetbare Substanz bei Handwärme, mit der gute Abdrücke und Abgüsse eines Gebisses z. B. erhalten werden können. Diese plastische Masse riecht stets nach Alkohol, aber es sind Jahre notwendig, so viel davon zu entfernen, daß die Substanz wieder bröcklich wird. Der letzte Rest Alkohol wird erst bei gelinder Wärme entfernt, eine bei ca. $135^{\circ}$ äbnlich der Malysäure schmelzende Harzsäure zurücklassend.

Aber auch andere organische Lösungsmittel wie Aether, Petroläther, werden von der Kolophoniumsubstanz absorbiert und hartnäckig festgehalten. Als ich eine wässerige Lösung der $\gamma$-Natronharzseife mit Aether ausschüttelte, um dadurch eine, den klebrigen Charakter der Harzseife bedingende Substanz zu entfernen, bemerkte ich eine über das gewöhnliche Maßs hinausgehende, nehr als reichiiche Absorption des Aethers. Die aus einer deraitigen ätherhaltigen Lösung mittelst Salzsäure făllbare $\gamma$ Pininsäure wurde in eigentümlichen bizarr gestalteten Gebilden von weicher Konsistemz, ohne flüssig oder plastisch $\mathbf{z u}$ sein, erhaiten, die ich als: "kristallisierte" kolloide Lösungen - so paradox dies auch klingen mag - bezeichnen möchte. Namentlich die Petroleumkohlenwasserstoffe, speziell die des tiefsiedenden Petroläthers neigen zur Bildung derartiger Substanzen.

Auch die ätherhaltige Substanz braucht viele Monate, um nur die Hauptmenge des Aethers zum Verdampfen zu bringen. Bei jedem erneuten Durchreiben tritt wieder Aethergeruch auf. Die schlieblich hinterbleibende Substanz $(\gamma$-Pininsäure) hat ibren Schmelzpunkt auf 85 bis $87^{\circ}$ erhöht. Das MiBlingen der Alkoholbehandlung veranlaBte $T s c h$ ir $c h$, die Behandlung seiner mit Ammoniumkarbonat erbaitenen Substanz mit Petroläther zu versuchen, um die oben geschilderten "Schmieren" $7 u$ entfernen. Es ist auf S. 652 und 653 seines grobzügig angelegten Werkes interessant zu lesen, wie hierbei die angewandte Substanz in Form firnisartiger Schmieren erneut und wie es scheint mehr als durch Alkohol zerflob.

Diese und andere noch $z u$ besprecliende Beispiele zeigen die Mannigfaltigkeit der kolloiden Erscheinungen der Kolophoniumsubstanz, die sich nicht mit der Aufnahme ganz bestimmter Mengen Wasser und Kohlenwasserstoffe begnügt, sondern noch befähigt ist, in diesem kolloiden Zustande weitere Mengen anderer organischer Verbindungen $z \mathrm{u}$ absorbieren und festzuhalten. Dadurch erklärt sich zum groben Teil die dieser Substanz anhaftende Rätselhaftigkeit.

Es geht aber auch daraus mit Sicherheit hervor, daf für das Kolophonium und die sich dâvon ableitenden Harzsubstanzen die Anwendung der sonst bei, organischen Arbeiten üb. lichen L.ösungsmittel, namentlich bei höherer Temperatur, nicht am Platze ist, da man stets gewärtig sein mul, neue Substanzen zu erhalten, die den Gang der Untersuchung erschweren und die Natur der ursprïnglichen vollständig verdecken. Ja selbst eine über das Maß hinausgehende Verdünnung mit destilliertem Wasser kann die Umwandlung einer bei ca. $100^{\circ}$ schmelzenden Substanz in eine solche bei $120^{\circ}$ schmelzend veranlassen. Konzentriertes Alkali, z. B. zehnprozentige Sodalosung, läBt die kolloiden Harzsubstanzen meist unverändert, nicht aber verdünnte. Erwärmt darf nur dann werden, wenn man des beabsichtigten Effekts sicher ist, wie $z$. B. bei der Bildung der $\gamma$ Natronharzseife. Kurz, jede einfachste Operation, deren Wirkung man nicht genau kennt, bedarf zur Feststellung eines eingehenden Studiums, wobei die geringste Veränderung, z. B. das Auftreten einer opalisierenden Trubung beobachtet und in Betracht gezogen werden mub. Erst auf dieser Grundlage sind die Harzsubstanzen willig und folgen den Richtungen, die zu gehen man thnen zuwies. Denn die Kolophoniumsubstanz ist in doppeltem Sinne eine fliebende. Nicht nur, dab sie meistans gewaltsam gezhungen wird, den lebenden Pflanzenorganismus fließend za: verlassen, auch ihre innere Bewegungsfahigkeit kormmt nicht eher zur Ruhe, bis eir den fossilen Harzen, wie z. B. dem Bernstein, ähnlicher Zustand erreicht ist.

Durch die Anwendung zweier, für geworhnlich nicht in den wissenschaftlichen chemischen und pharmazentischen Laboratorien besonders geachteter Substanzen, Petroleum und kalkhaltiges Leitungswasser - ist es mir moglich gewesen, den Veränderungen der Kolophoniumsubstanz in ihrem gesetzmäbigen Verlaufe $z u$ folgen und damit einige Klarheil uber dessen wirkliche Natur zu erlangen. In neuerer Zeit gesellt sich dazu die von Ts chirch gefundene Methode der Bleiazetatfällung in alkoholischer Losung (Tschirch S. 653), die er rach dem Fehlschlagei der Alkoholkristallisation und der Petrolätherbehandlung benutzt, um aus der durch Ausschütteln mit Ammoniumkarbonat erhaltenen Substanz zwei hochschmelzende Harz- 
säuren, die $\alpha$ - und die $\beta$-Abietinsäuren, die also für isomer angenornmen werden, voneinander zu trennen.

Die $\alpha$-Abietinsäure (Schmelzpunkt 143 bis $\left.150^{\circ}\right)$ wird hierbei als weiBer voluminosser und gelatinöser. Niederschlag gefällt, der anfangs gelblich, sich erst später bei genügendem Zusatz des Fällungsmittels in den weißen Niederschlag umwandelt. Es unterliegt keinem Zweifel, daP Tschirch ein Gemenge unter Händen hatte, und neben der von ihm angenommenen $a$-Abietinsäure noch $\gamma$-Pininsäure durch die stark verdünnte Ammoniumkarbonatlösung aus" geschüttelt wurde, die ihrerseits allerdings eine rein weiße und voluminöse Fällung in alkoholischer Lösung durch eine ebensolche basisch essigsaure Bleiazetatlösung erleidet. Aus dem alkoholischen bleihakigen Filtrat wird die $\beta$ Abietinsäure (Schmelzpunkt $145-153^{\circ}$ ) als lockeres gelbliches Pulver erhalten. Diese Angaben haben mich veranlast, einer Reihe von Substanzen die $\beta$-Vorzeichnung zu geben $(\beta$ Pininsäure, $\beta-K L w$ - Harzsäure), die ihren sonstigen Eigenschaften zufolge, zu denen vor allem die stark saure Natur und die damit verbundene hohe Seifenzahl (Tschirch S. 660) gehört, den Substanzen der $\alpha$ - Reihe, also speziell der von Ts chirch gefundenen $\alpha$ - Abietinsäure, gleichzusetzen wären. Da aber diese Substanzen Umwandilungsprodukte sind, die erst während der 600 maligen Ausschüttelungen entstehen, können sie auch nicht isomer sein und ihre Bezeichnung Abietinsäure muß zukünftig einer anderen Platz machen. Man vergleiche die Schwierigkeiten, die Tschirch (S. 654) beschreibt, um einigermakien für die Analyse geeignetes Material zu erhalten, welches, nach seinen Angaben (S. 654), bei längerem Stehen in alkoholischer Lösung wieder braune Schmieren absetzte, und bewies, dab das kolloid geburidene Wasser noch nicht entfernt war und wie ich zuversichtlich behaupten kann, aut diese Weise aus den vorher entwickelten Gründen überhaupt nicht zu entfernen ist. Dementsprechend sind die analytischen Daten der Formel $\mathrm{C}_{19} \mathrm{H}_{28} \mathrm{O}_{2}$ angepaßt (S. 655) oder deren Unsicherheit (S. 659) durch die Anwendung des Wortes ,,scheint" zum Ausdruck gebracht. Es liegt mir durchaus fern, die mühevollen Arbeiten Tschirch's herunterzusetzen, namentlich da es ihm durch die Anwendung der Bleiazetatfallung gelang, die Grundlage zu schaffen, auf der allein es mir möglich war, die Existenz dreier Körperklassen zu begründen, die, allerdings nicht in den hochschmelzenden $\alpha-, \beta$ - und $\gamma$-Abietinsäuren, sondern in den tiefschmelzenden $\alpha$-, $\beta$ und $\gamma$-Pininsäuren $z u$ suchen sind, die, wie ich annehme, durch Aufnahme kolloid gebundenen Wassers aus ersteren entstanden zu denken sind. Aber es ist ein Irrtum Tschirch's, diese von ihm als $\alpha$ - und $\boldsymbol{\beta}$-Abietinsäure bezeichnieten Substanzen als präexistierend in Verbindung mit einem indifferenten Harz anzune ${ }^{\circ}$ men, vielmehr hat sich als unzweifelhaft erwiesen, dab diese erst und $z$ war in Form der tiefschmelzenden kolloid gebundenes Wasser enthaltenden $\alpha$ - und $\beta$-Pininsäure aus der Grundsubstanz des Kolophoniums, der $\gamma$-Pininsäure, bei jeder alkalischen Behandlung, ja schon durch gröBere Mengen kalkhaltigen Wassers gebildet werden. Der experimentelle Beweis ist leicht durch die Anwendung reiner und einheitlicher $\gamma$-Pininsãure zu erbringen, wenn diese in ätherischer Lösung nach Tschirch mit Ammoniumkarbonatiösung ausgeschüttelt wird. Die daraus mittelst Salzsäure gefällten Harzsäuren gehen zum groben Teil im Gegensatz zur $\gamma$-Pininsäure durch Leitungswasser in Lösung und werden daraus durch geringe Mengen Salzsäure als bei 100 bis $110^{\circ}$ schmelzende Substanzen gefält. In weiterer Verfolgung dieser Beobachtung habe ich drei Gruppen von Harzsubstanzen avfstelien können :

$$
\begin{aligned}
& \gamma \text {-Pininsüure } \\
& \text { Hauptkolophoniumsubstanz; } \\
& \text { Schmelzpunkt } 75-76,5^{\circ} \\
& \alpha-K L w \\
& 100-105^{\circ} \\
& \beta \cdot \mathrm{KLW} \\
& 120-1234
\end{aligned}
$$

Auch die durch Behandlung mit Alkohol von allen Nebenbestandteilen und somit auch von den leichter löslichen $\alpha$ - und $\boldsymbol{\beta}$-Pininsäuren befreite Malysäure ${ }^{5}$ ). die als die in der Mitte zwischen der das HöchstmaB kolloid gebundenen
Wassers enthaltenden $\gamma$-Pininsäure und der als absolute Kolophoniumsubstanz keinen kolloiden Zustand mehr darstellenden $\gamma$-Abietinsăure

b) Tschirch, Die Harze und die Harzbehalter (1916), 661. 
stehende Etappe zu betrachten ist, hat Tschirch $150 \mathrm{mal}$ ausgeschüttelt (Tschirch S. 651), in der Hoffnung einer schnelleren Abnahme der durch Ammoniumkarbonat extrahierbaren Harzsäuren. Da aber nach dem 150. Male der Ausschüttelung diese Abnahme nicht eintrat, wurde der Versuch als aussichtslos aufgegeben.

Es kann nach allem wohl als sicher gelten, dab die durch Ammoniumkarbonat aus dem Kolophonium oder der durch Reinigungsprózesse, wie Behandeln mit 70 prozentigem Alkohol erhaltenen Malysäure oder mit Hilfe der $\gamma$-Natronharzseife darstellbaren $\gamma$-Pininsäure. abscheidbaren Harzsâuren, die sogenannten $\alpha$ und $\beta$-Abietinsăuren, nicht vorgebildet im Kolophonium und dessen daraus erbältlichen Präparaten enthalten sein können, sondern sich erst während des Ausschüttelns bilden und daher mit der später zu beschreibenden, als absolute Kolophoniumsubstanz bezeichneten $\gamma$ Abistinsäure nicht isomer sein können.

Um letztere zu erhalten, schüttelt $T$ schirch die restierende ätherische Kolophoniumlösung (Tschirch S. 657) mit einprozentiger Sodalösung aus. Es ist bezeichnend, dah er dazu nur 20 Ausschüttelungen nötig hat, weil die $\gamma$-Pininsäure, die er in Form ihrer Natronharzseife in Lösung bringt, nicht erst gebildet werden muB, sondern vorhanden ist. Es ist selbstverständlich, dab sich hierbei ebenfalls wieder die als Umwandlungsprodukte der $\gamma$-Pininșäure bezeichneten Harzsäuren: die $\alpha$ - und $\beta$-Pininsăure bilden werden, natürlich nur in viel geringerer Menge, da nur eine 20 malige Behandlung vorliegt. Ein von Tschirch unter $\mathrm{Zu}$ hilfenahme von alkalischer Bleiazetatlösung ( Ts chirch S. 658) angestellter Versuch verlief ergebnislos, ist aber kein Beweis für dio Abwesenheit gelblich ausfallender geringer. Mengen der $\beta$-Verbindung. Zudem ist der Versuch diesmal in anderer Weise, nämlich in alkalischer Lösung ausgeführt worden, während früher die alkoholischen Lösungen der freien Harzsäuren zur Anwendung kamen; das scheint mir nicht gleich zu sein. Zwar hat das Studium der Einwirkung von Chlorkalziumlösung oder stark kalkhaltigen Leitungswassers auf eine $\gamma$-Natronharzseifenlösung unzweifelhaft ergeben, daB der Prozes der Umbildung bzw. der Fällung durch Metallsalzlösungen ein äußerst komplizierter ist und letzten Endes zu einer petroleumlöslichen Kalkverbindung führt, die nach der Behandlung bei einer bei ca. $340^{\circ}$ liegenden Tempe:atur die Fähigkeit erlangt, Kohlenwasserstoffe in Form einer kautschukähnlichen Substanz kolloid zu binden. Aehnlich werden die Bleisalze sich verhaiten. Sicher ist, daß die Einwirkung alkoholischer Bleiazetatlosung auf die alkoholischen Loosungen der Harzsubstanzen nicht durch den einfachen Vorgang eines leicht- und schwerlöslichen Bleisalzes erklart werden kömnen. Es sind mir Falle bekannt, daß anfängliche Fällungen bei geringem UeberschuB des Fallungsmittels wieder in Lösung gingen; ferner, daß aus wasseriöslichen, bei ca. $120-124^{\circ}$ schmelzenden Substanzen solche bei $205-210^{\circ}$ in Form einer aus braunschwarzen Tropfen gebildeten Tropfenkette schmelzende übergeführt werden können. Die Versuche darüber sind erst begonnen und bilden wieder ein Kapitel inbezug auf die Umbildungsvorgänge, denen die Harzsubstanzen infolge ihres wechselnden Verbaltens, einmal in Form kolloider Lösungen oder als absolute Harzsubstanzen, aufzutreten, unterworfen sind. Es scheint nach allem dem Bleiazetat, in alkoholischer Lösung auf die freien Harzsäuren einwirkend, die Fähigkeit zuzukommen, den Harzsubstanzen ihre kolloide Fähigkeit zu nehmen. Diese Andeutungen sollen nur die Unsicherheit der Bieisalztrenmung vor Augen führen und nicht als endgiltige Ergebnisse zu betrachten sein. Da nun auf Grund meiner früheren Auseinandersetzungen die Umwandlung und Ueberführung der kolloid gebundenes Wasser entbaltenden Kolophoniumsubstanz oder dieser gleichkommenden $\gamma$-Pininsäure in die als absolute Harzsăure anzune? mende $\gamma$-Abietinsäure chemisch betrachtet darauf hinauskommt, das gebundene $W$ isser zu entfernen, so würden möglicherweise $z u$ der nur unter groben Verlusten ausführbaren Alkoholbehandlung die Bleiazetatfâllung in alkoholischer Lösung treten.

Nochmals sei darauf hingewiesen, dab die ätherische Kolophoniumlösung nach 600 maliger Ausschüttelung mit Ammoniumkarbonat, bei der schon reichlich braunen Schmieren bei nach. folgender Alkoholbehandlung auftraten, die erneute Sodaausschüttelung bei gleicher Nach. behandlung diese Schmieren abermals zeigte (Tschirch S. 657). Diese sind, wie ich schon oben auseinandersetzte, nur als Ausdruck der Widerstandsfähigkeit der Kolophoniumsubstanz zu betrachten, mit der diese jeder Störung ihrer kolloiden Natur entgegentritt.

Die Beimengung eines neutralen indifferenten Harzes gilt danach als abgetan. Dic durch Sodaausschüttelung erhaltene $\imath^{\prime}$-Abietinsäure schmilzt bei $153-154^{\circ}$; erst die durch 
Vakuumdestillation gereinigte zeigte den Schmelzpunkt $161 \%$.

Die nächstliegende Methode der Wasserentfernung böte unzweifelhaft eine Behandlung bei höherer remperatur. Aber es ist ja bekannt, dab die Kolonhoniumsubstanz sich lieber zerstören läBt und Harzol bildet (Tschirch S. 674-675), ehe sie in glatter Weise das kolloid gebundene Wasser abgibt. Dieses muk demnach in einer Form, wenn auch nicht chemisch, gebunden sein, die ihre ureigene Natur als kolioide Lösung zum Ausdruck bringt, deren energische Störung von einem Zerfall des Moleküls begleitet ist. Es wird demnach die Störung eines auf physikalischer Grundlage beruhenden Verhältnisses zwischen der chemisch als absolute anzusehenden Kolophoniumsubstanz und Wasser den Zerfall auch in chemischer Bezieliung nach sich ziehen. Vielleicht ist in diesem Verhältnis das Wesen der kolloiden Erscheinungen an der Kolophoniumsubstanz als neuer Typus einer festen kolloiden Lösung zu suchen. Eine scheinbare Ausnahme macht in dieser Hinsicht das Verhalten des Kolophoniums oder der $¥$-Pininsäure, wenn diese in Form ihrer Petroleumlösung destilliert, also erhitzt wird.

Kolophonium wie die $\gamma$-Pininsäure zeigen eine große Löslichkeit in Petroleum in der Kälte, die derjenigen des Zuckers in Wasser gleichkommt. Schon ein Teil Kolophonium mit $1-1 \frac{1}{2}$ Teilen Petroleum in einer Reibschale gut verrieben, bildet eine sirupartige Lösung, die sich leicht durch Nessel von dem geringfügigen Rückstand, der für große und durchsichtige Stücke ca. 1 Proz. beträgt, abfiltrieren läßt. Durch Nachwaschen anfangs mit Petroleum, später mit Petroläther, läßt sich der Rückstand (KR), dessen Menge bis zu 86 Proz. steigen kann, zwischen Zeitungspapier abpressen und bildet dann ein leichtes grauweißes oder sclrwach gelblich gefärbtes Pulver.

Die Petroleumlosung, selbst die mit Petrolather, bleibt bei Anwendung großer Kolophoniumstücke für einige Zeit klar: Aber nach längerem Stehen und namentlich bei erneutem Petrolätherzusatz scheiden sich geringe Mengen des KR-Rückstandes ab, als Ausdruck eines unentwegt fortschreitenden Uniwandlungsprozesses, der sich durch die Zunahme des petroleumunlöslichen KR-Rïckstandes kennzeichnet und kontrollieren läBt. Die Destillation des Petroleunfiltrats effolgt in normaler Weise bis eixa $300-310^{\circ}$. Danach tritt sogenanntes Sruatzen ein infolge explosionsartig entweichen- den Wassers. Zeigten die Destillate bis zu dieser Temperatur ein mehr dern Petroleum ver. wandtes und hinsichtlich der Farbe wie dieses ein farbloses Ansehen, so tritt nach $310-320^{\circ}$ bis etwa $350^{\circ}$ ein dickflüssigeres, gelbgefärbtes Oel auf, das ich in meiner ersten Abhandlung darüber mit "leichter Balsam" bezeichnete ${ }^{6}$ ). Die Entstehung dieses Destillats ist mit steigender Temperatur verbunden, die eine Höhe von $360^{\circ}$ erreichen kann. Dann fällt das Thermometer trotz gröBerer Wärmezufuhr und geht bis auf $310^{\circ}$ herunter, wobei ein dem Harzöl ahnliches schwerflüssiges bis zähes Destillat erhalten wird, der "schwere Balsam".

Aus dem leichten Balsam scheiden sich nach monatelangem Stehen bei Zimmertemperatur konzentrisch gruppierte bis $1 \mathrm{~cm}$ lange spiebige Kristalle $a b$, die oftmals und nach jahrelangem Stehen die ganze Flüssigkeit durchsetzen. Aber auch der schwere Balsam zeigt dieselben Kristallisationserscheinungen nach jahrelangem Stehen. Nach Entfernung des Destillats durch Ablaufen, Ausbreiten der Kristalle auf Papier usw., kann der anhaftende Rest durch vorsichtige Behandlung mit Petroläther entfernt werden. Die chemische Zusammensetzung ergab bei der Analyse: 74,52 Proz. C, 8,81 Proz. H, 16,66 Proz. Sauerstoff. Der Schmelzpunkt der Substanz lag bei $150-159^{\circ}$ und glich der Bildung einer wirklichen Lösung, die allmählich in den unteren Teil des Schmelzpunktröhrchens abflob; ein Erstarren derselben durch Abkühlung und Stehen wurde bisher nicht wahrgenommen. Die Substanz löst sich leicht in Alkohol bei gelindem Erwärmen urid kristallisiert aus der. Lösung beim Erkalten wieder in großen spiebigen $\mathrm{Kri}$ stallen. Wird aber die Losurrg gekocht und dabei etwas eingedampft, so tritt eine Scheidung in zwei Schichten ein. Aus der unteren, also schwereren, infolge ihres Gehalts an Kohlenwasserstoffen auch öligerer Abscheidung, kristallisiert die $\gamma$-Abictinsäure in kleinen farblosen, bej $161-163^{\circ}$ schmelzenden Blättchen.

Die großen spiebigen Kristalle sind demnach als eine Kohlenwasserstoffverbindung der $\gamma$ Abietinsäure anzusehen, die so wenig stabil ist, daß schon eine kochende alkoholische Lösung deren Zersetzung herbeifühit. Von einer chemischen Verbindung kann daher kaum gesprochen werden, auch der Vergleich mit dem Kristallwasser reicht nicht aus, da in diesem Fall die geschmolzene Substanz wieder erstarren müBte. Daher ist deren Zustandekominen nur

6) Chem. Revue uber die Fett- und Harzindustrie 1914, S. 38. 
auf der Grundlage der kolloiden Fähigkeit zu erklären, die bei den Harzsubstanzen auch gegenüber den Kohlenwasserstoffen des Petroleums in die Erscheinung tritt und zwar diesmal in Forn ausgesprochener Kristalle, die als der Typus "kristallisierender " kolloider Lösungen aufzufassen sind. Während also die Bindung des Wassers als Dispersitätsmittels nur zu der amorph auftretenden Form, dic ich als $\gamma$-Pininsäure beschrieb, führt, bleibt im analogen Falle bei der Verwendung hochbindender Kohlenwasserstoffe die kristallisierte Form der $\gamma^{\prime}$. Abietinsäure nicht nur erhalten, sondern tritt in gesteigertem MaBe hervor. Der Vorgang ist in beiden Fallen derselbe und immer auf die Fähigkeit der wasserfreien Kolophoniumsubstanz z:rückzuführen, einen Teil seines Dispcrsitătsmittels in Form einer festen kolloiden Lösung zu fallen und festzuhalten, diesmal in Form ausgesprochener Kristalle.

Beim Erwărmen treten wirkliche L.ösungen ein, die nicht mehr zur Kristallisation zu bringen sind. Auch sonst ist die Bildung der Kristalle in ein gewisses Dunkel gehüllt, nicht nur durch die lange Zeit der Abscheidung, sondern vor allem durch die Unmöglickeit, deren Bildung direkt durch Erhitzen der Kolophoniumsubstanz mit einer üher $300^{\circ}$ siedenden Petroleumfraktion zu bewirken. Das gleiche gilt von dem $\mathrm{Er}$ hitzen einer Kolophon-Petroleumlösung, aus der die Fraktion bis $310^{\circ}$ entfernt wurde, am RückfluB kühler.

Beide Substanzen, die $\because \cdot$ Abjetinsäure und deren Kohlenwasserstoffverbindung, sind unbegrenzt, hinsichtlich der Zeit unzersetzt aufzubewahren. Durch Verkochen einer alkoholischen Lősung mit fünfprozentiger Sodalösung bis zur vollständigen Entfernung des Alkohols bildet sich die $\gamma$-Natronharzseife, aus der die $\gamma$-Pininsäure vom Schmelzpunkt 75-76 abgeschieden werden kann. Da nun umgekehrt die $\gamma$-Pininsăăre, wie das Kolophonium, bei der Destillation ihrer Petroleumlösung wieder in die $y-$ Abietinsäure bzw. deren Kohlenwasserstoffverbindung zurückverwandelt wird, so ist dadurch nicht nur die Identităt der $\gamma$-Pininsăure mit der Grundsubstanz des Kolophoniums gegeben, sondern auch das Verhältnis zwischen $\gamma$-Pininsăure und $\gamma$-Abietinsäure als einer kolloid gebundenes Wasser enthaltenden Substanz zu seiner absoluten, d. h. in ihrem kolloiden Zustande gestörten und daher wasserfreien Substanz einwandsfrei festgestellt. Leider sind die Ausbeuten nur sehr gering und betragen nur mehrere Prozente der angewandten Kolophoniumsubstanz:
Der gröBte Teil wird, wie ich schon andeutete, in Harzöl zersetzt.

Pininsăure und $\gamma$-Abietinsäure sind dem nach als die üußersten Punkte einer Reihe zu betrachten. die mit der $\gamma$-Pininsäure als wasserreichste das HöclistmaB kolloid gebundenen Wassers enthaltenden Substanz beginneud über die Sylvinsäure und Malysăure, mit geringerem Wassergehalt zur wasserfreien, ihrer kolloiden Fähigkeit beraubten $;$-Abietinsăure führi. Da also in der $\gamma$-Pininsäure die kolloide Fähigkeit in bezug auf Wasser am stärksten ausgebildet ist, und in der $\gamma$-Abietinsäure den Wert von Null besiłzt, so künnen die drei Säuren, da deren Existenz in chemischer Beziehung vielleicht mit Reciht als șelbstandige Säuren bestritten werden kann, als kolloide Zustände, als Polymerisationswirkungen der Kolophoniumsubstanz in bezug auf Wasser bezeichnet werden.

$\gamma$-Pininsăure $=1$. kolloider Zustand oder 1. Polymerisationswirkung ;

Sylvinsäure $=2$. kolloider Zustand oder 2. Polymerisationswirkung;

Malysäure $=3$. kolloider Zustand oder 3. Polymerisationswirkung;

$\gamma$-Abietinsăure $=$ absolute wasserfreie Kolophoniumsubstanz.

Allen diesen Substanzen ist auBerdem die gemeinsame Reaktion zu eigen: 1 . in alkobolischer Lösung durch eine alkoholische Bleiazetatlosung in Form eines weißlen, anfangs gelatinösen Niederschlags gefällt zu werden, dann 2. durch verdünnte, aber überschüssige Ammoniaklösung gelatinös zu erstarren, infolge Bildung der $\boldsymbol{\gamma}$-Ammoniakharzseife.

Aus dem Vorhergehenden lassen sich folgende Punkte mit Sicherheit als feststehend herausheben :

1. Das Kolophonium des Handels ist der neue Typus einer festen kolloiden Lösung;

2. Weder hochschmelzende Harzsäuren noch ein indifferentes Harz sind darin nachweisbar;

3. Die Grund- und Hauptsubstanz ist die bei $75-76^{\circ}$ schmelzende $\gamma$-Pininsäure, zugleich das Höchstmaß kolloid gebundenen Wassers enthaltend und daher als erster kolloider $Z$ u stand bezeichnet;

4. Durch wasserentziehende Substanzen, wie Alkohol, ferner durch Erhitzen, wie bei der Destillation mit Petroleum, wird der kolloide Zustand infolge Wasserabgabe zerstört, womit zugleich teilweise eine Zerstörung der Substanz auch in chemischer Hinsicht eintritt; 
5. Dabei wird in geringer Menge die absolute, $d$. h. keine Spur kolloid gebundenen Wassers enthaltende Kolophoniumsubstanz, die $\gamma$ Abietinsäure rom Schmelzpunkt $161-163^{\circ}$ gebildet :

6. Durch Behandlung mit Alkalien, wie Soda oder Ammoniak, werden daraus die $\gamma$-Alkaliharzseifen gebildet, aus deren wässeriger Lösung die Ausgangssubstanz, die $\gamma$-Pininsäure, zurückgebildet und somit der erste kolloide Zustand wieder hergestellt wird;

7. Wie Wasser, kann die absolute Kolophoniumsubstainz auch Kohlenwasserstoffe des Petroleums kolloid binden;

8. Es ist jedoch hervorzuheben, daB die groß kristallisierende und bei $150-159^{\circ}$ schmelzende Kohlenwasserstoffverbindung der $\gamma$-Abietinsäure eine Umwandlung, wie die als neuer Typus einer festen, kolloiden Lösung bezeichnete Kolophoniumsubstanz, nicht erfährt und es daher fraglich erscheint, diese der letzteren gleich zu setzen.

9. Dagegen können diejenigen Kohlenwasserstoffe oder Aetherverbindungen, die unter gewissen Umständen aus kolloid gebundenes Wasser enthaltenden Harzsubstanzen entstehen, deren bizarre Ciestaltung oftmals das Aussehen von Kristallen vortäuschen, vielleicht als, $\mathrm{kri}$ stallisierende" Kolloide bezeichnet werden.

10. Mit Alkohol bilden die meisten kolloid gebundenes Wasser enthaltenden Harzsubstanzen sirupartige lïsungen, die von Tschirch') als Harzschmieren bezeichnet werden.

11. Die Möglichkeiten zwischen $\gamma$-Pininsăure und $\gamma$-Abietinsäure stehender kolloider Zustände sind natürlich unbegrenzt und nur mit Rücksicht auf die bereits bekannten, nach feststehenden Methoden entstehende Sylvin- und Malysäure beschrankı worden.

7) Tschirch, Die Harze und die Harzbehalter (1906), 3 .

\title{
Die Industrie der vegetabilischen Leime im Kriege.
}

\author{
Von Ernst Stern (im Felde).
}

(Fingegangen am 27 . Juli 1917.)

Eine Anzahl bedentsamer Industriezweige, oie wesentlich auf kolloidchemischer Giundlage aulgebaut sind, besitzer das gemeinsame Kenuzeichen, daß $z u$ ihren unentbehrlichen Grundstoffen Eiweib oder Kohlehydrate gehören, - sie sind also abgesehen vom Kautschuk und den Zelluloseprodukten wichtige Reprăsentanten der angewandten organischen Kolloidchemie; es gehören hierzu die Industrie der plastischen Massen, die Galalithindustrie und vor allem dic Industrie der Klebstoffe. Der Umstand, daß diese Industriezweige gerade die wichtigsten Grundstoffe der menschlichen Enälırung zu gewerblichen Zwecken benutzen, hat es mit sich gebracht, daß sie sehr bald, nachdem das Problem der Sicherstellung der Volksernahrung in seiner ganzen Bedeutung erfaßt worden war, von einschneidenden MaBnahmen betroffen wurden. Es war naturtich vor allem erforderlich, alle EiwejB- und Kohlehydratquellen für Ernährungszwecke und für die. Futtermittelgewinnung möglichst vollstăndig nutzbar zu machen und wenn mogilich für die fehlenden Kraftfuttermittel neue Quellen zu erschliebew. Das Verfahren der Lufthefegewinnung vou D elbrü $c k$ ist ein hervorragendes Ergebnis dieser Bestrebungen: ferner haben sie dazu gefuhrt, daB man aus den Rohstoffen der Industrie der tierischen Leime - Knochen und Hautabfallen - eir neues Eiweiß-Kraftfuttermittel herstellt. Man wird in der Tat zu der Frage geführt, ob die Klebstoffindustrie überhaupt ejne Fxistenzberechtigung hat in einer Zeit, in der die Volksernihrung and die Beschaffung von Waffen und Munition der oberste Leitsatz für jede industrielle Arbeit sein mub. Diese Frage soll in bezug auf die Industrie der vegetabilischen Leime näher geprält werden.

Die Industric der vegetabilischen Leime oder Pflanzenleime ist eine wesentlich aul kolloidchemischer
Erkenntnis aufgebaute Industrie; ihre charakteristische Eigenart ergibt sich aus einem vergleichenden Hinweis auf die Industrie der tierischen leime. Die Ge. winnung der tierischen Leime geht im wesentlichen in der Weise vor sich, daß mani aus dem passend vorbereiteten Leimgut (Hautabfalle, Knochenschrot) eine Leimbrühe gewinnt, die geklärt und in Vakuumapparaten eingedampft wird. Das erkaltete Konzentrat bildet eine Gallerte von 30-50 Proz. wasserfreicm Leim, die in Leimschneidemaschinen za dïnnen Tafeln zerschnitten wird. Diese Tafeln werden schlieblich einem Trockenproze日 unterworfen, der lhren Wassergehalt auf etwa 10 Proz. buingi. Dieser Tafelleim ist das ibliche Handelsprodukt. Um ithn für den Gebrauch fertig 2 u machen, muB man natürlich den letzten Teil der fabrikatorischen Herstellung umgekehrt durchlaufen, denn man läBt den Leim erst in Wasser etwa 24 Stunden aufquellen und stellt dann durch gelindes Erwarmen die gebrauchsfertige Leimbrühe her. Vor einiger Zeit bat schon $W$ ilhelm Ostwald in der "Kolloid-Zeitschrift" auf die unwirtschaftliche Seite dieser Arbeitsweise aufmerksam gemacht; denn es wird tatskichlich ein erheblicher Teil der Herstellungskosten von dem TrockenprozeB beansprucht, also von eitien Teil des Verfahrens, der nachher für den Gebrauch des Leimes wieder rückgängig gemacht werden muB.

Im Gegensatz hierzu bringt die Pflanzenleimindustrie ihre vielseitigen Produkte zum aberwiegen den Teil gebrauchsfertig in den Handel. Sie stelit, kolloidchemisch ausgedritckt, haltbare Emulsionskolloide industriell her. Der Grad der Stabilität dieser Emulsionskolloide ist in Verbindung mit den leimtechnischen Eigenschaften ein wichtiger Gradmesser für die Erzeugnisse dieser Industrie. 